\title{
Mindfulness y compasión. Cambio de paradigma en las relaciones interpersonales. Una perspectiva fenomenológica
}

\author{
Dr. Miguel Ibáñez Ramos \\ Director de Mindfulness-Research, Zen-Teacher of EWS \\ Autor correspondencia: miguelibez@gmail.com
}

Recibido: 12/10/2018; Aceptado: 23/11/2018; Publicado: 30/01/2019

Resumen: La posibilidad de cambiar el paradigma cognitivo fruto de las prácticas que desarrollan la atención plena, no sólo afecta a la reactividad frente a las circunstancias y a la disminución de la automatización emocional que repercute en el sufrimiento personal, sino que favorece el desarrollo de una dimensión diferente de la experiencia relacional e interdependiente. Esto constituye un nuevo marco interpretativo del sufrimiento, que se traduce en un cambio activo de conducta que llamamos compasión. En nuestra reflexión presentaremos desde la perspectiva de la fenomenología el potencial integrador y saludable de los ejercicios de desarrollo de la atención plena, en especial, de la dimensión del amor compasivo como dimensión intersubjetiva de la experiencia y su potencial transformador de las relaciones y de la ética.

Palabras Clave: Mindfulness; Meditación; Amor Compasivo; Ética; Fenomenología.

\begin{abstract}
The possibility to change the cognitive paradigm as a result of mindfulness practices, not only affects the reactivity to the circumstances as well as the reduction of emotional automation, that triggers personal suffering, but promotes the development of a different dimension of the relational and interdependent experience. This brings us to a new interpretive framework of suffering, wich transforms into a new active behaviour that is called compassion. This reflection presents, from the perspective of phenomenology, the integrating and healthy potential of mindfulness's exercises, particularly, its dimension of love and kindness and compassion as an intersubjective dimension of the experience as well as its transforming potential of relationships and ethic.
\end{abstract}

Key words: Mindfulness; Meditation; Loving Kindness; Ethic; Phenomenology.

\section{Introducción}

Hablar de mindfulness y compasión en un contexto de educación para la salud implica dos aspectos fundamentales: Por un lado, el conocimiento preciso de la fenomenología de la práctica de la atención plena y de las prácticas de compasión, tanto en el análisis del conjunto de procesos que intervienen en la misma, como en sus consecuencias a nivel personal e intersubjetivo. Por otro lado, es también necesaria la descripción de un marco hermenéutico adecuado a nuestra mentalidad y lo más amplio posible que nos permita comprender los procesos que configuran la experiencia y que también nos sirva para comunicarla de forma clara y adaptada al aprendizaje por parte de otros. Hoy día son muchos los estudios que avalan la práctica de mindfulness $[6,4,25]$ y en ellos se constatan cambios tanto a nivel funcional como estructural en nuestro cerebro. En esto vemos un aspecto transformador de la relación con la experiencia cotidiana al tiempo que se muestran cambios en diversos parámetros de la salud física, psicológica e interrelacional que condicionan el bienestar [25]. Aunque a nivel metodológico, a veces, se hace necesario separar ambos aspectos (mindfulness y 
compasión) en realidad, y este es nuestro punto de partida, son inseparables y han de cultivarse de forma conjunta, ya que ambos se condicionan mutuamente. Este planteamiento constituye nuestro marco hermenéutico en este trabajo, visto desde la fenomenología cognitiva y las ciencias contemplativas.

Como indica Auxiàs Cebolla, [9] (p. 74), mindfulness se puede conceptualizar en tres vertientes: como una técnica de meditación susceptible de entrenar (sin embargo, la práctica de mindfulness va más allá de la meditación en cuanto tal); como proceso psicológico o estado mental; como constructo psicológico susceptible de definir y operativizar. Los elementos que forman parte de este constructo son muchos y nada fáciles de encasillar. En él intervienen múltiples aspectos y los podemos abordar, tanto desde los procesos mentales implicados, como desde las consecuencias individuales y relacionales derivadas de ellos [17] (p. 5).

Desde el punto de vista de la tradición budista, sati (mindfulness) se refiere originariamente a la capacidad de recordar [1] (p.66) pero va más allá de la mera memoria, tiene que ver con lo que permite que se sostenga un contenido de experiencia en la conciencia, y es un aspecto de la Cuarta Noble Verdad: "el óctuple camino para superar el sufrimiento". En este contexto, sati, forma parte de los tres últimos factores del camino óctuple y se sitúa entre el recto esfuerzo (samma vayama), que tiene que ver con el discernimiento de los pensamientos turbadores para impedir que proliferen y al tiempo sostener los pensamientos constructivos que aumentan las emociones positivas; y la recta concentración (samma samadhi) que conduce a la experiencia de la mente apaciguada y estable, que permite una experiencia no dual de la realidad a través de la absorción (dhyana). Ahora bien, para pasar de uno a otro se requiere la capacidad de mantener el foco de atención sostenida en el proceso de formación de la experiencia, y esto es sati o atención plena, lo que permite dilatar y estabilizar la conciencia en el presente que es donde aflora el flujo de la experiencia, momento a momento. Esto es lo que denominamos "momento cognitivo" el instante en que se forma la experiencia. El ejercicio de esta cualidad nos permite dirigir el foco de la atención a la experiencia en el cuerpo y las sensaciones, las emociones, y las actividades de la mente, así como los pensamientos y los elementos que constituyen las vivencias de las cosas, con aceptación. [1] (pp.37-38).

El término aceptación implícito en la definición generalizada de la práctica de mindfulness está íntimamente unido a la ecuanimidad (upekkha) que no tiene que ver con resignación, sino más bien con una actitud sabia que dirige la acción con el espíritu sereno (con las emociones en equilibrio) en el proceso de interacción con los seres y circunstancias con las que se establece una relación en plena consciencia, momento a momento. Como fruto de la comprensión de la impermanencia de todo fenómeno, expresión de la dimensión dinámica de la existencia, esta relación se hace progresivamente más desvinculada de la imagen mental creada de lo vivido.

Esta aceptación profunda resuena en la experiencia consciente, en función de las circunstancias de las que emerge dicha experiencia: compasión (karuna) ante el sufrimiento que deriva de la ignorancia, amor bondadoso (metta) ante el reconocimiento de la unidad de todos los seres sensibles; júbilo o dicha (mudita) ante la felicidad existencial compartida. Estos tres elementos junto con la ecuanimidad son considerados el fundamento de la ética (acción correcta) vinculada a la práctica de la atención plena y se denominan los cuatro inconmensurables. [12] (p. 94 y 242).

En este breve párrafo hemos intentado sintetizar el marco de la tradición budista entorno a mindfulness y compasión, sobre el que luego se ha desarrollado la práctica tal y como la conocemos en su versión secular y aplicaciones clínicas, pero es muy importante no perder de vista el trasfondo fenomenológico, que según la tradición budista implica un detalle muy significativo: lo que constituye la realidad no son "entidades autónomas en sí mismas" que se relacionan unas con otras, sino fenómenos (procesos energéticos y cognitivos) que aparecen ante una conciencia y se hacen objeto de conocimiento, condicionados por multitud de circunstancias que los hacen posible.

Así pues, en donde budismo y fenomenología coinciden es en una visión de la existencia dinámica, apareciendo y desapareciendo en nuestra conciencia según las circunstancias, momento a momento. Ahora bien, la realidad es a la vez el conjunto de fenómenos y lo que los hace posibles. Esto significa que lo que constituye un fenómeno en sí es la interdependencia con múltiples circunstancias y el ámbito en el que se producen, una de las cuales es la conciencia en la que se hace 
presente el fenómeno. Esta dimensión relacional de la existencia es el significado esencial de la impermanencia (anicca) y de la ausencia de ente sustantivo (anatta) cuya ignorancia de ambos constituye la fuente de todo sufrimiento, ya que nuestra mente se aferra a los fenómenos como si fueran fijos y constantes [13] (pp. 292-293).

En el presente artículo pretendo desarrollar de forma estructurada los elementos constitutivos de la práctica de mindfulness y compasión tanto en su dimensión subjetiva como intersubjetiva en relación con la transformación de la experiencia. Con este fin, tendré presente tanto la introspección (experiencia en primera persona) como de la fenomenología en diálogo con la ciencia, ya que como dice J. Searle: "el coste de despreciar la filosofía consiste en cometer errores filosóficos" [5] (p. 97). “La fenomenología es la filosofía de la experiencia. Según Husserl el principio de reflexión filosófica requiere de quien lo emprende la actitud de un principiante absoluto, esto significa, libre de prejuicios" [3] (p. 163) [11]. Esta actitud coincide con el fundamento de la práctica de mindfulness de permanecer presente en la experiencia tal y como sucede, así Bhikkhu Bodhi comenta "la tarea inicial de sati consiste en mantener el 'registro desnudo de los hechos observados' tan libre como sea posible de las elaboraciones conceptuales distorsionantes" [16] (p. 72).

La fenomenología es la ciencia que estudia cómo el mundo se hace cognición consciente por su cualidad intencional [24] (p. 40) y sus consecuencias vitales. En frase de Acebes, "tanto el mundo como los objetos se muestran de forma constante en «formas de aparición» diferentes. De ahí surgía la investigación del cómo de las multiplicidades fenoménicas, esto es, de esas formas de aparición, con vistas a la constitución de una cosa y del mundo como unidad de sentido y validez" [3] y añado: objeto de lenguaje, ya que es el lenguaje el que da sentido a la percepción y posibilita un universo compartido, de ahí la importancia de la experiencia en segunda persona [17] (p. 5). La fenomenología y posteriormente la hermenéutica nos dan las claves de cómo el intercambio de energía e información con el medio implícito en toda forma de vida (vivencia) se convierte en experiencia autoconsciente, la cual en función de su dimensión relacional adopta un sentido compartido que puede resultar una experiencia integradora o todo lo contrario. Así pues, tenemos una sucesión de procesos que configuran la experiencia cognitiva que se resume en: vivencia-experiencia-autoconscientedimensión relacional.

Es esta maleabilidad en la formación y emergencia de la experiencia en la conciencia la que ha hecho que las tradiciones contemplativas descubran la posibilidad de entrenar los procesos mentales constructores de la misma en un sentido integrador de la experiencia, lo que redunda en un aumento de la felicidad [20].

Las tradiciones vinculadas a la práctica de la atención plena, en especial el budismo, nos han insistido en este carácter de ejercicio, de entrenamiento, de cultivo (Bahvana) de ese constructo psicológico complejo denominado mindfulness. Pero al hilo de esta afirmación nos preguntamos: ¿qué es exactamente lo que se ejercita y en qué sentido se hace? y más importante aún ¿cuáles son las condiciones y la motivación que fundamentan y sostienen este ejercicio?

A mi entender, en estas dos preguntas se resume el título de nuestro artículo, ya que como comenté no entiendo la práctica de mindfulness y la de compasión como dos cosas separadas, sino como consecuencia necesaria la una de la otra. La práctica de mindfulness conlleva la transformación del paradigma cognitivo, y por ende, de la experiencia de una manera global, pero centrada en la manifestación de la existencia impermanente y dinámica como un "continuum" de momentos cognitivos interrelacionados en los que se hace presente un conjunto de fenómenos, que emergen al hilo de la percepción consciente. Nuestro sistema cognitivo es en cierto modo paradójico: a nivel temporal nos ofrece una sensación de continuidad, linealidad y permanencia y a nivel espacial nos presenta una radical diferenciación y fragmentación de la realidad formando entes enmarcados en sus límites y características.

De estos dos aspectos nace el apego (y con él el sufrimiento). En resumen, el apego surge del tipo de relación, que de forma espontánea "mi sistema" establece con la representación experiencial del momento cognitivo antes descrito, fruto del filtro interpretativo particular de mi mente, dotándole a esa representación de entidad sustantiva (que no tiene) y juzgándola como apetecible o rechazable bajo los parámetros de supervivencia implícita en mi individualidad. La mayor parte del 
sufrimiento psicológico se fundamenta en las expectativas creadas ante circunstancias, cosas o personas y no en la realidad tal cual se presenta. Por eso es tan difícil la aceptación sincera. De la relativización de los procesos cognitivos y la apertura a la experiencia de lo impermanente nace progresivamente una aceptación compasiva que supera el sufrimiento individual y sirve de motivación para procurar que todo ser se vea liberado del mismo sufrimiento. Este es el nuevo paradigma interrelacional.

Como vemos en esta pequeña descripción nuestro sistema por defecto está tan orientado a preservar la supervivencia que nos hace caer en la confusión al mezclar la mente y sus procesos con la realidad. Este estado es denominado por la tradición ignorancia esencial (avidya) y la práctica de mindfulness es la puerta a la sabiduría (pañ̃ã) [24] (p. 51), que supera la ignorancia y posibilita responder con compasión.

\section{Mindfulness como fenómeno cognitivo relacional.}

\section{1 ¿Qué es exactamente lo que se ejercita y en qué sentido se hace?}

Hoy en día, cuando hablamos de mindfulness nos referimos tanto a una serie de prácticas, como a la capacidad que se desarrolla por medio de las mismas. Dada la enorme variedad de posibles prácticas orientadas a ejercitar la facultad que denominamos atención plena, los científicos, a la hora de valorar los resultados específicos de una investigación deben concretar lo más posible el tipo de práctica al que refieren el estudio, así como los períodos de tiempo dedicados y de esta manera establecer un marco objetivo con el que correlacionar el resto de parámetros estudiados. Así pues, siguiendo el marco fenomenológico propuesto por Luz y cols. [17] las prácticas que consideremos en este trabajo son principalmente la atención focalizada (FA) y la atención abierta (OM) [15]. Pero no todo acaba ahí. La facultad a la que afecta la práctica es algo más complejo que la mera atención y tiene que ver con el núcleo de la emergencia de la experiencia cognitiva, el momento cognitivo, que se forma en tres fases:

Con frecuencia, en este esquema se habla de exterior (estímulos) e interior (todo lo demás). Bajo esta perspectiva late el sentido de que nosotros somos un algo diferente dentro de un mundo de elementos que nos afectan y con los que reaccionamos (a esto lo denominamos el supuesto entitativo). En realidad, todo depende del punto de vista en que situemos la dinámica interrelacional, ya que todo está interactuando con todo, también nosotros, de forma que lo que percibimos como "entidades" no son sino el resultado del encuentro entre cierta energía modulada y el procesamiento cognitivo en nuestra conciencia [13] (p. 293).

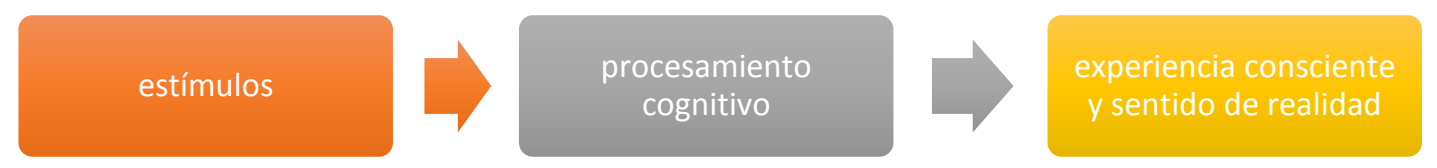

Figura 1 Fases que forman el momento cognitivo

Veamos paso por paso. Los estímulos están limitados por nuestro sistema perceptivo corporal (nuestros cinco sentidos a los que la tradición budista añade la propia mente) y es tan importante esta afirmación que nada de lo que conocemos puede ser objeto de ciencia experimental si no la reducimos (aunque sea con ayuda de la tecnología) a una percepción sensorial. Este es el primer filtro que clasifica la interacción energética según los sentidos que interactúan con ella. A este nivel E. Gendling lo denomina "sensación sentida" y es la resonancia del flujo de energía con el que interactúa nuestro cuerpo (redes neuronales activas), que también son un flujo de energía codificada sobre la que se construye la auto-consciencia como proceso meta-cognitivo [13] (p. 290).

Desde la fragmentación multi-estimular del entorno, el proceso cognitivo es un proceso de unificación, de agrupamiento, de construcción de engramas neuronales en frase de J. Monserrat [16] que pueden asociarse con procesos de interacción repetidos y esto se realiza, como indica $\mathrm{A}$. Damasio,[7] (pp. 110-112), por capas a distintos niveles en nuestra estructura neuronal cortical y subcortical, de manera que una suma de momentos cognitivos sucesivos y de múltiples estímulos 
recibidos y filtrados se convierten en formas, objetos, colores, sonidos, seres vivos etc. con los que nos relacionamos. De ahí que el hecho relacional condicione la forma resultante.

Por último, algo en nosotros nos dice que esa experiencia es real o no; aunque a veces cuesta distinguirlo (cuando tenemos una pesadilla, por ejemplo). Lo que determina el "sentido de realidad" es la experiencia sensorial en relación a la integridad de mi cuerpo y de ahí el "color emocional" de la experiencia. A estos tres elementos descritos les siguen estados de conciencia que condicionan la respuesta conductual:
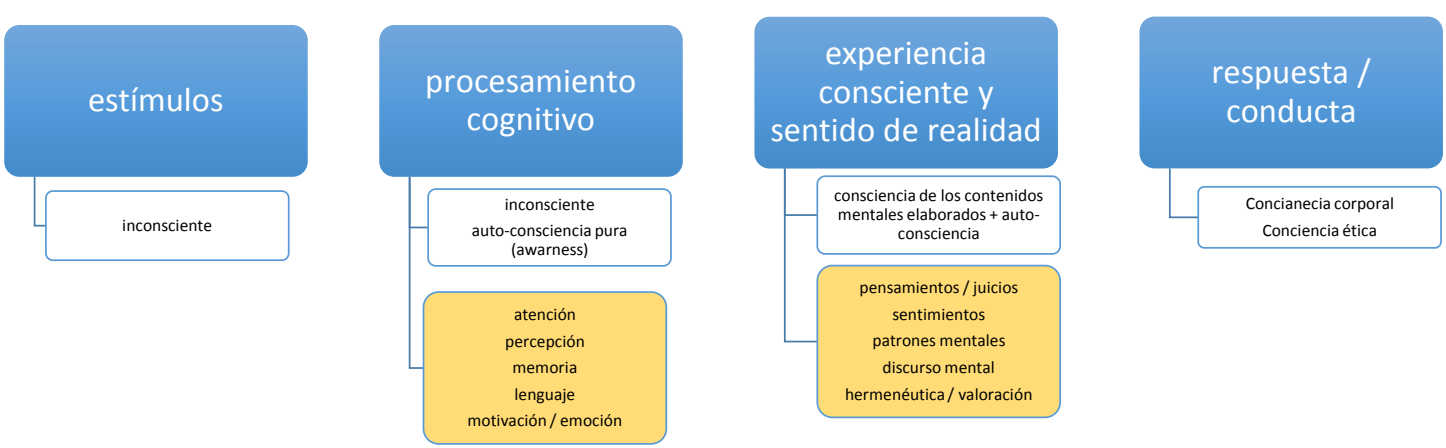

Figura 2 Estados de conciencia que condicionan la respuesta conductual

El flujo de los momentos cognitivos sucesivos es tan rápido que es difícil distinguir estos tres pasos. Por ello, el resultado final sería imperceptible sin un elemento vital del procesamiento cognitivo: la fijación en la memoria modulada por la atención que permite que enlacemos un momento con el siguiente, teniendo presente el anterior y así sucesivamente, haciendo de la experiencia una sensación de algo fijo y continuo [7], (p. 120).

En su libro ¿Qué es la mente? El gran erudito budista (gueshe) Lati Rimpoché describe el momento cognitivo con estas palabras: “En el budismo, la mente no se concibe sólo como una reserva general de información o como un mecanismo del cerebro, sino como momentos individuales de conocimiento cuyo continuo constituye nuestro sentido de conocer" [20] (p. 15).

Una vez secuenciada la experiencia, la mente la compara con otras, la valora y la retiene, generando un bucle que se puede sostener en el tiempo, realimentando la respuesta emocional una y otra vez, y consolidando la auto-afirmación: el ego. Así pues, nos encontramos con dos elementos que condicionan nuestra experiencia: la dificultad de ver la realidad tal y como es, ya que sólo aparece como fenómeno en el momento cognitivo y la tendencia constructora de la mente que genera tantos pensamientos que acabamos identificándolos con la realidad. Ambos elementos se potencian mutuamente de forma que a mayor construcción y autoafirmación del ego, mayor dificultad para comprender la realidad tal y como es.[20] (pp. 58-59).

De este proceso nacen el prejuicio, la visión parcial, el apego, la resistencia al cambio, las creencias, la intolerancia, la sensación de verdad absoluta, y en último término, el sufrimiento psicológico. La experiencia y el sentido de realidad se ven nublados por el funcionamiento de cada mente modulada por cada cuerpo con lo que conlleva de reactividad emocional ante los acontecimientos.

Una de las aportaciones más importantes de las ciencias cognitivas ha sido ofrecernos un marco de interpretación multidisciplinar para entender los fenómenos cognitivos. Las dos corrientes más relevantes son la denominada "la mente corporeizada" (Embodied Mind) representada por G. Lakoff y Mark Johnson y la de "espacios conceptuales" de P. Gärdenfors [14] p.117. Ambas ponen de manifiesto que los procesos cognitivos son los responsables de nuestra experiencia de lo real y por tanto son constructores de lo que denominamos el mundo.

La práctica de mindfulness, tal y como la conocemos hoy fue incorporada de la tradición budista, especialmente Theravada, por Jon Kabat-Zinn, con una finalidad terapéutica, para aliviar el 
sufrimiento de enfermos con dolor crónico creando el método MBSR (Mindfulness Based Stress Reducction) y a partir de ahí se desarrollaron numerosos programas terapéuticos (MBCT, ACT, MSC, CCT. DBT etc.) A la hora de describir los elementos que confluyen en este fenómeno cognitivo transformador y con consecuencias terapéuticas nos encontramos con un debate sobre qué es realmente mindfulness, que dependiendo del marco hermenéutico en el que se sitúe, pone el acento en unos aspectos u otros, así como en la consideración psicológica de los factores implicados en el desarrollo de esta capacidad y sus consecuencias. En algunos casos esto ha dado origen a cierta confusión y debate entre diversos expertos [16] (p. 51). Cuando se ha intentado dar respuesta a la efectividad terapéutica de la práctica, así como a la investigación sobre la misma, ha sido necesario diseñar metodologías experimentales que estén dentro de los marcos de investigación homologados por la ciencia, para lo cual se ha hecho necesario concretar lo más posible tanto el tipo de práctica y sus características, no sólo en cuanto a las instrucciones a seguir, sino también el seguimiento concreto de los procesos psico-corporales implicados en ella, de forma que se unifiquen los criterios a la hora de hacer una investigación. En segundo lugar, ha sido necesario determinar los elementos medibles en relación con la práctica determinada y su efecto en relación a tiempo, situación socio-familiar, contexto vital y circunstancias personales etc. No cabe duda que este proceso, en muchos casos, resulta reduccionista y deja fuera del marco de investigación numerosos aspectos de la práctica [17] (p. 6).

Es un hecho la enorme profusión de estudios científicos publicados en revistas y editoriales de gran prestigio $[6,8,23]$ sin embargo, sólo hace unos años se ha iniciado una reflexión en busca de un marco hermenéutico adecuado para acercarse a la comprensión de este fenómeno cognitivo que se presenta como un ejercicio que de alguna manera transforma la experiencia. De ahí la importancia de la fenomenología y las ciencias cognitivas.

Nuestro punto de vista filosófico se sitúa un paso antes del debate descrito y pretende esclarecer el marco hermenéutico que nos permita entender el proceso que se sigue en la práctica y el porqué de su transformación. En este caso se trata de un fenómeno cognitivo, ya que la práctica de la atención plena influye en el modo como experimento la realidad, y a partir de ahí, en cómo me relaciono con las circunstancias que concurren en ella [22].

Veamos los elementos que constituyen la práctica y su relación con la génesis de la experiencia, antes descrita, siguiendo la matriz fenomenológica propuesta por A. Luz y colls. [17] (p. 8). La práctica fundamental del desarrollo de la atención plena es la meditación [15] (pg. 45). Aunque esta palabra puede ser muy ambigua [24] (pp. 48-49), la meditación orientada a la atención plena se caracteriza por ejercitar de modo intencional la atención focalizada (FA) como base estable (samatha)para la atención abierta $(\mathrm{OM})$, dilatando el campo de la conciencia cada vez más hasta abarcar la totalidad de la experiencia con aceptación. Eso significa, penetrar en la interrelación (vipassana) de todos los fenómenos disolviendo las construcciones mentales que fijan la atención a pequeñas partes de la interacción de los mismos.

Como hemos visto anteriormente, nuestros procesos cognitivos crean un cúmulo de interferencias en el campo de la conciencia que nos dificultan ejercer la atención plena y simple a la experiencia presente. Por ello, necesitamos crear las condiciones más favorables posibles para que emerja este tipo de conciencia abierta a la experiencia. La primera de estas condiciones es el silencio y la quietud. No hablamos sólo de ausencia de diálogo, hablamos de un proceso de silenciamiento que comienza por la totalidad del cuerpo. Nuestro cuerpo se mueve constantemente al unísono con nuestra agitación mental [7] (pg. 172). Pero, cuando nuestra atención es intensa, el cuerpo naturalmente se detiene. Esta relación mente-cuerpo, puesta hoy de manifiesto por la neurociencia cognitiva, constituye la base de la práctica de mindfulness. En efecto, la práctica de atención plena es ejercitar la presencia atenta en la experiencia en el instante en que ésta surge en la conciencia desde el cuerpo. Y esto es más fácil hacerlo en estado de quietud y fracciones mínimas de tiempo (una respiración, por ejemplo). Ahora bien, nuestra vida no transcurre en este estado de forma continua, por lo que es fácil perder esta presencia en cuanto nuestra mente acompaña el movimiento y el movimiento exige la evaluación de la mente ante las circunstancias, de ahí surgen los otros ejercicios: meditación caminando, y yoga dinámico o conciencia dinámica corporal. En estos otros ejercicios se 
cambia el foco y en lugar de la postura en quietud sostenida por la respiración, la atención se dirige al flujo de sensaciones y experiencias cambiantes del cuerpo en movimiento, momento a momento. Y por último están las prácticas de compasión en las que el foco se sitúa en la relación con los demás y las resonancias emocionales derivadas del modo de percibir la relación.

Así pues, a medida que el ejercicio se repite, la atención es más sostenida y es más fácil percibir cuándo la conciencia se hace eco de un juicio o pensamiento sobre la experiencia y cuándo está simplemente en la experiencia tal y como es. En esta diferencia está la clave de la práctica.

Este ejercicio sostenido posibilita un proceso de desautomatización emocional reactiva. Esto no significa que desaparecen las emociones, sino que se experimentan sin el vínculo identitario que es el que nos dispara los pensamientos y juicios que sostienen el bucle emocional auto-identificativo. No es igual sentir ira por una circunstancia que sentirse ofendido a nivel personal y alimentar la ira pensamiento a pensamiento al margen de las circunstancias que lo provocaron. En esta dinámica, es precisamente, donde la práctica mindfulness enlaza con la compasión.

\subsection{La compasión y la dimensión relacional del momento cognitivo.}

La cognición es una función que emerge del proceso vital y cuyo objeto no es otro que codificar la interrelación con el entorno en orden a sostener la dinámica inestable que es la vida misma en forma de individuo. La práctica de mindfulness, orientada a la visión y experiencia de la realidad tal y como es, nos muestra que la existencia es fluida y cambiante, momento a momento, (anicca o impermanencia de todo fenómeno) lo cual contrasta con la sensación de nuestra cognición habitual, que muestra las cosas fijas y sustantivas, aunque finitas. ¿Qué es lo que hace estable y fija a la realidad, que en sí misma es permanente cambio? La respuesta es la cualidad del momento cognitivo. Pero, la fijación mental del mismo es sólo adaptativa, no real. Por eso el momento cognitivo es relacional, se da según circunstancias conjuntas, en un instante determinado y puede cambiar al siguiente. Al cultivo de esta atención consciente al surgimiento del momento cognitivo, momento a momento, responde el concepto de sabiduría (pañña), que no es otra cosa que penetrar en la dimensión relacional del surgimiento de la experiencia, reconociendo su impermanencia. En definitiva, experimentar la realidad tal y como es: fenoménica y vacía de contenido sustancial.

La dimensión relacional del momento cognitivo no es percibida sólo a nivel objetivo, como si el sujeto fuera un observador separado, sino que la experiencia sólo es posible en la interacción total en la que el sujeto cognitivo es una circunstancia condicionante más que surge en el instante mismo de la cognición (pratitya samutpada, es un concepto que describe el origen interdependiente de todo fenómeno y su surgimiento condicionado, lo que constituye la base de su carencia de sustancialidad independiente). Si no se incluye el sujeto en la experiencia, estaríamos en una experiencia descorporizada o como dice Varela citando al filósofo Th. Nagel "una visión desde ninguna parte" [24] (p. 52). Ahora bien, el sujeto también está condicionado y es insustancial en el sentido antes descrito. Esta es la clave para la comprensión de la raíz de todo sufrimiento que nace de considerar la realidad y el sujeto como algo fijo y parcelado, a la par que confundir nuestros procesos mentales con la experiencia misma de lo real, como ya hemos indicado [1] (p. 85).

Todo momento cognitivo que emerge de la interacción conlleva simultáneamente el posicionamiento afectivo-emocional de nuestro sistema-individuo en el entorno: la orientación relacional que está condicionada. Es decir, cognición, emoción y conducta (respuesta) son inmediatos, el problema es que, dependiendo del grado de conciencia, muchas veces, la conducta es reactiva y automática, es decir, movida no por la comprensión de la realidad en su profundidad sino por el condicionamiento de los factores mentales sumidos en la ignorancia, que hemos descrito como mediación para la supervivencia básica.

La compasión es, precisamente, la conducta que nace de la comprensión (sabiduría) de la interdependencia de todo en la sucesión de los momentos cognitivos. No existen realidades independientes o aisladas. Cualquier forma de sufrimiento afecta a la totalidad y cualquier individuo puede aminorarlo en su respuesta relacional derivada del momento cognitivo. La compasión es actuar según la verdadera naturaleza interconectada de la realidad. Por eso en sánscrito la palabra compasión (Karuna) tiene la misma raíz que la palabra acción (Karma). La compasión en el contexto 
de la atención plena no es tanto un sentimiento o un buen propósito, cuanto la recta manera de actuar derivada de la comprensión sabia de la realidad con el fin de aminorar el sufrimiento.

Al escuchar la palabra compasión, en nuestros idiomas tendemos a relacionarla con el sentimiento de lástima o pena ante la desgracia ajena. No cabe duda de que el sufrimiento personal o ajeno mueve en sujetos normales una serie de mecanismos neuronales que provocan empatía, como sentimiento de identificación y resonancia con el dolor ajeno. Como bien indica J. Soler [9] (pp. 4950), el núcleo de la compasión está relacionado con la aspiración natural de todos los seres a ser felices y hoy día conocemos los mecanismos de las neuronas espejo. Ahora bien, ¿qué entendemos por felicidad? Podemos pensar que lejos de ver la felicidad como la emoción de alegría es lo más parecido a la homeostasis, es decir, desarrollo natural de la existencia en ausencia de sufrimiento, momento a momento.

Aunque el lenguaje nos obliga a describir primero un proceso y luego otro, en la experiencia no ocurre así. De la conciencia modulada por la atención plena emerge la compasión y el amor bondadoso en el mismo instante en que se va despegando de las actividades mentales constructoras (mindfulness) y al mismo tiempo, ya que somos seres relacionales, la conducta movida por la compasión aumenta la experiencia de atención plena. De este bucle de retroalimentación nace el concepto de "humanidad compartida" sobre el que se apoyan las prácticas de compasión [19, 21]. La humanidad compartida nos vincula a todos los de nuestra especie, tanto en el sufrimiento como en los recursos para superarlos, por ello, la capacidad de ampliar el foco en la acción relacional fruto de las prácticas de mindfulness y compasión nos abren a un nuevo paradigma interpersonal.

\section{La compasión, un nuevo paradigma ético.}

La interrelación es la característica del flujo de la existencia. El ser es dinámico y cambiante en su dimensión fenoménica. A medida que el individuo es más complejo su interrelación está determinada por un número mayor de factores implicados. Por ello, al igual que el sistema cognitivoperceptivo unifica los estímulos y los procesa, la zona ejecutiva de nuestro sistema determina la respuesta interactiva, es decir, el intercambio de energía con esa parcela de realidad identificada mentalmente. La atención plena a la realidad sin apego a los filtros interpretativos nos permite modular la experiencia reconociendo en ella una resonancia íntima de amor gozoso (metta), ante la realidad que se manifiesta armónica, o bien experimentando compasión (karuna) ante la emergencia de la experiencia de dolor como hemos indicado arriba. Ambas actitudes brotan de la visión profunda resultado de la atención plena, cuando ésta se ve libre del enredo mental y se mantiene en la ecuanimidad. El problema es que este discernimiento o visión clara no es frecuente, ya que enseguida se mezcla con nuestro mundo mental (juicios, prejuicios, opiniones, emociones implicadas etc.) que, no sólo condiciona la visión, sino que además abre las puertas a impulsos y reacciones emocionales automáticas, con frecuencia gestadas en nuestro dolor personal y que están muy lejos de ser las más sabias y adaptadas a las circunstancias.

Por este motivo, junto con la práctica de la atención plena, cabe el cultivo específico de la compasión y el amor bondadoso como ejercicio de aceptación de la experiencia presente y cauce facilitador de la conducta en respuesta a las circunstancias. Desde la práctica de la atención plena toda respuesta debe nacer de la compasión como intención de erradicar el sufrimiento, tanto personal como colectivo y medioambiental. Esto es un cambio en la experiencia surgida en el momento cognitivo y precisamente este cambio permite que la relación con la experiencia se vaya transformando.

En la tradición filosófica occidental desde Aristóteles hasta Heidegger, incluida la tradición juedeo-cristiana, la ética se considera un paradigma principalmente normativo. En frase de Aristóteles en su Ética, capítulo XXXIV, “la ética es una acción que está de acuerdo con un principio recto". Es decir, se considera primero el análisis intelectual sobre el bien, luego se universaliza, para en tercer lugar elaborar un código de acción que deberá aplicarse en cada caso o circunstancia determinada. La tradición de la atención plena, en cambio, plantea un nuevo paradigma relacional, cuyo punto de partida es la sabiduría. Pero no una sabiduría de conocimientos adquiridos (que sería más erudición) sino la sabiduría originaria, la que está libre de construcciones mentales, prejuicios, 
sesgos culturales etc. Se trata de la sabiduría que emana de la experiencia directa de la naturaleza relacional de todo ser, vivida momento a momento. Esta sabiduría encuentra en la compasión que emana de forma natural un medio hábil para actuar de forma adecuada a las circunstancias y cuya orientación básica no es otra que aliviar, aminorar el sufrimiento en todas las direcciones de las interrelaciones de la acción [24] (p. 286).

En este sentido la atención dirigida a la experiencia se fortalece con la atención centrada en la respuesta de acción y sus cualidades como un todo continuo. La tradición de la atención plena sostiene un ejercicio principalmente no-activo, es decir, que más que hacer algo, consiste en evitar la implicación en los mecanismos automáticos de reacción. Este evitar, tampoco es una acción contraria, que sería contraproducente, sino que la mera consciencia del surgimiento de estos mecanismos y la no implicación en ellos los detiene poco a poco ya que se ponen de manifiesto su trasfondo de ignorancia y sufrimiento y se acoge con compasión.

No cabe duda de que el ejercicio sostenido de esta práctica, cambia el modo de relación con las circunstancias y con ello cambia radicalmente la experiencia y aumenta la sensación de bienestar [24] (p. 691). El presupuesto para incorporar esta práctica de ejercicio de la compasión a la terapia es según Germer que "una terapia exitosa es la que cambia la relación del paciente con su sufrimiento" [10] (p. 31). Por ello, el nuevo paradigma ético no consiste en cambiar unas normas por otras, sino en cultivar la presencia en la experiencia, incluida la acción y la respuesta relacional, buscando en todo momento el bienestar personal y de todos los seres implicados. Esto concuerda con el viejo adagio de la regla de oro presente en numerosas tradiciones: "trata a los demás como te gustaría ser tratado" pero entendiendo en ello el pleno desarrollo de la sabiduría y la comprensión que anulan la ignorancia y suprimen el sufrimiento.

Este cambio de paradigma ha sido estudiado en aplicación a la relación médico paciente y el síndrome de burnout por el Dr. García Campayo y colls. [4] y su estudio pone de relieve que, si por un lado, la práctica de la meditación de atención plena tiene efectos directos sobre la experiencia de estrés y aminora los síntomas del mismo y aumenta el bienestar de los profesionales, las prácticas de compasión fortalecen este efecto y añaden otro muy relevante, que es la facilidad de comunicación con el paciente y la implicación personal en esta relación.

\section{Conclusiones}

Hoy día son muchos los profesionales y muy diversos los ámbitos en los que se desarrolla la práctica de mindfulness. Podemos decir que se ha producido un salto cualitativo del ámbito contemplativo al mundo secular sin precedentes. La razón principal de la extensión de esta práctica, no cabe duda, que es su eficacia probada, su repercusión en la salud mental y su dimensión transformadora de la relación con la experiencia.

Ahora bien, se trata de una práctica que requiere ejercicio constante y que se va fortaleciendo a medida que se se sostiene en el tiempo.

En este trabajo hemos abordado la dimensión relacional de la práctica de mindfulness y compasión. En primer lugar, como consecuencia directa de transformación de la experiencia. En segundo lugar, como reflexión hermenéutica aplicada a una nueva visión de la realidad y en tercer lugar, como nuevo paradigma ético y sus consecuencias en las relaciones interpersonales.

Esta reflexión, constituye sólo un esbozo y un punto de partida de las muchas conclusiones que pueden derivarse de la experiencia de la práctica de la atención plena.

Un aspecto que ha puesto de relieve el estudio de los fenómenos asociados con la práctica de la atención plena y la compasión es la necesidad de un trabajo interdisciplinar que permita una visión multidimensional de los aspectos implicados en la misma. Desde el punto de vista de la fenomenología, un campo que a mi entender está todavía por desarrollar y que es imprescindible para profundizar en la dimensión relacional de mindfulness, es precisamente, la experiencia en segunda persona, lo cual implica, en primer lugar, conocer los mecanismos subyacentes a la verbalización de la experiencia al ser compartida (fenomenología del lenguaje, psicolingüística, lingüística estructural etc.), así como el marco hermenéutico que posibilita que esa experiencia 
conduzca a un proceso integrador que denominamos comprensión íntima o insight, que transforma el sentido de la existencia.

Quedan todavía numerosos aspectos en las tradiciones contemplativas que pueden aportar diversas dimensiones a la práctica permitiendo que se establezca de forma más continua y compatible con cualquier circunstancia de la vida. Y por último, notamos un campo de investigación que se ha puesto de relieve en el estudio de esta práctica que es la relación cuerpo-cerebro-mente-consciencia. El trasfondo filosófico todavía impreciso en la descripción de los elementos constitutivos de esta interacción no podrá ser resuelto desde la mera especulación, sino incorporando en el proceso de conocimiento la experiencia en primera persona junto a los otros planos de experiencia estudiados desde diversas ciencias sobre un consenso conceptual y terminológico que permita la objetivación del estudio y el análisis en tercera persona.

\section{Referencias Bibliográficas}

1. Analayo, B. Satipatthana. Los orígenes del Mindfulness, Barcelona: Siglantana. 2018

2. Anuruddhacariya, B. A Manual of Abhidharmma. Abhidammattha Sangaha. Kuala Lumpur, Malaysia: Buddhist Missionary Society. 19794.

3. Acebes, R. Subjetividad y mundo de la vida en Husserl y Merleau-Ponty (Historia, cuerpo y cultura). Consultado el 2 de septiembre 2018. [Internet] (1995). Tesis Doctoral UCM: disponible en: Webs.ucm.es/BUCM/tesis/19911996/H/2/AH2009401.pdf

4. Amutio-Kareaga, A.; García-Campayo, J.; Delgado, L.C.; Hermosilla, D.; Martínez-Taboada, C. Improving Communication between Physicians and Their Patients Through Mindfulness and Compassion-Based Strategies: A Narrative Review. Journal of Clinical Medicine, 2017, 6, 33. Disponible en Pubmed: https://www.ncbi.nlm.nih.gov/pubmed/28304333 .

5. Capra, F. Las conexiones ocultas. Implicaciones sociales, medioambientales, económias y biológicas de una nueva visión del mundo. Barcelona: Anagrama, 2003.

6. Chiesa, A.; Serretti, A. Mindfulness-Bassed Stress Reduction for Stress Management in Healthy People: A Review and Meta-Analysis, Journal of Alternative and complementary Medicine [internet] vol.15. n.5. 2009 pp.593-600. Disponible en pubmed: https://www.ncbi.nlm.nih.gov/pubmed/19432513

7. Damasio, A. Y el cerebro creó al hombre. ¿Cómo pudo el cerebro generar emociones, sentimientos, ideas y el yo? Barcelona: Destino, 2010.

8. Davidson, R. El perfil emocional de tu cerebro. Barcelona: Destino. 2012.

9. García-Campayo, J.; Cebolla, A.; Demarzo, M. La Ciencia de la Compasión. Más allá de mindfulness. Madrid: Alianza Editorial. 2016.

10. Germer, CH.K.; Siegel, R.D.; Fulton, P.R. Mindfulness y Psicoterapia. Bilbao: Desclée de Brower. 2015.

11. Guerrero del Amo, J.A. ¿Es la fenomenología la solución al problema de la conciencia?, [internet] Thémata. Revista de Filosofía № 46 (2012) pp.: 271-279. Disponible en: http://institucional.us.es/revistas/themata/46/art_22.pdf

12. Harvey, P. El budismo. Barcelona, Cambridge University Press, 1998.

13. Ibáñez, M. Aspectos transformadores de la experiencia humana a la luz de la práctica de la atención plena. ÉNDOXA: Series Filosóficas, n.o 42, 2018, pp. 285 -308. UNED, Madrid. [En prensa]

14. Iglesias, L. Ciencia cognitiva. Introducción y claves para su debate filosófico. [internet] Trabajo de investigación Universidad de Navarra. 2006.2 Disponible en: http://www.unav.es/gep/TesisDoctorales.html

15. Kabat-Zinn, J. La práctica de la atención plena. Barcelona: Kairós, 2005.

16. Kabat-Zinn, J.; Williams, M. G.; Mindfulness. Su origen, significado y aplicaciones. Barcelona: Kairós, 2017.

17. Lutz, A.; Jha, A.P.; Dunne, J. D.; Saron C. D.; Investigating the Phenomenological Matrix of Mindfulness related Practices from a Neurocognitive Perspective, Am Psychol. [internet] (2015). 70(7). 632-658.

18. Montserrat, J. Engramas Neuronales y teoría de la mente, Pensamiento 57 (228):177-221 (2001). Disponible en: https://docplayer.es/14433919-Engramas-neuronales-y-teoria-de-la-mente.html

19. Pommier, E.A. The compassion scale. (Dissertation), Austin TX: The University of Texas Austin, 2010.

20. Rimpoché, L. ¿Qué es la mente? La explicación del budismo tibetano. Novelda (Alicante): Dharma ed. 2001

21. Salzberg, S. A heart as wide as the world, Boston, Massachusetts: Shambala ed. 1997. 
22. Siegel, R.D. La Solución Mindfulness. Bilbao: Desclée de Brower.2012

23. Simon, V. “Mindfulness y neurobiología”, Revista de Psicoterapia, (2007) 66(67): 5-30.

24. Varela, F.; Thomson, E.; Rosch, E. De cuerpo presente. Las ciencias cognitivas y la experiencia humana. Barcelona: Gedisa. 19972

25. Wallace, B.A.; Shapiro, S.L. Mental Balance and Well-Being. Building Bridges Between Budhism and Western Psychology. Am Psychologist [internet] (2006) 61, (7), 690-701. Disponible en: https://www.sbinstitute.com/sites/default/files/mentalbalance.pdf

26. Wayment, H.A.; Bauer, J.J. Transcending self-interest: Psychological explorations of the quiet ego (159-169), Washington, DC, American Psychological Association. 2008.

(C) 2019 por los autores; Esta obra está sujeta a la licencia de Reconocimiento 4.0 Internacional de Creative Commons. Para ver una copia de esta licencia, visite http://creativecommons.org/licenses/by-nc-nd/4.0/. 